\title{
The influence of probiotics on individual fecal secondary bile acid levels: a two-case study of schizophrenic patients receiving an atypical antipsychotic drug
}

\author{
Yosuke Saito $^{1}$, Hiroyuki Nishimiya ${ }^{2}$, Yasue Kondo ${ }^{3}$, and Toyoaki Sagae ${ }^{1}$
}

${ }^{1}$ Yamagata Prefectural Yonezawa University of Nutrition Sciences, 6-15-1, Torimachi, Yonezawa, Yamagata, 992-0025, Japan; ${ }^{2}$ Public Interest Incorporated Foundation, Sekizen-kai Soga hospital, 148, Sogakishi, Odawara, Kanagawa, 250-0203, Japan; ${ }^{3}$ Medical Corporation, Ziundo hospital, 414-53, Sekimachiminami, Nerima, Tokyo, 177-0053, Japan

Corresponding author: Yosuke Saito, Master's, Yamagata Prefectural Yonezawa University of Nutrition Sciences, 6-15-1, Torimachi, Yonezawa, Yamagata, 992-0025, Japan

Submission Date: August 17th, 2017, Acceptance Date: November 25th, 2017, Publication Date: November 30th, 2017

Citation: Saito Y., Nishimiya H., Kondo Y., Sagae T., The influence of probiotics on individual fecal secondary bile acid levels: a two-case study of schizophrenic patients receiving an atypical antipsychotic drug. Functional Foods in Health and Disease 2017; 7(11); 849-858. https://doi.org/10.31989/ffhd.v7i11.384

\begin{abstract}
Background: Probiotics is used as a promising approach in the prevention and treatment of hypercholesterolemia. Modification of bile acid metabolism through the deconjugation of bile salts by microbial bile salt hydrolase $(\mathrm{BSH})$ is considered to be the core mechanism of the hypocholesterolemic effects of probiotics. Nevertheless, BSH activity is reported to be detrimental to the human host due to the generation of toxic secondary bile acids. Thus, the influence of probiotic intake on bile acid metabolism needs to be elucidated. We analyzed the bile acid levels and microbiota in human fecal samples after probiotic supplementation to assess the influence of probiotic intake on fecal bile acid levels. Two patients hospitalized for schizophrenia and dyslipidemia, receiving an atypical antipsychotic drug, were enrolled in this study (Subjects A and
\end{abstract}


B). Both subjects received Lactobacillus rhamnosus GG (LGG) for 4 weeks, and no probiotics for the following 4 weeks. Fecal samples were collected at baseline and after 4 and 8 weeks.

Results: Conjugated bile acids may be modified by indigenous intestinal bacteria into unconjugated bile acids and secondary bile acids through deconjugation reactions by BSH activity and the subsequent $7 \alpha$-dehydroxylation pathway, respectively. In the fecal microbiota from Subject A, the relative abundance of Bifidobacterium increased after LGG supplementation (30\%49\%). Most Bifidobacterium and Lactobacillus strains that colonize mammalian intestines may report BSH activity, and in general bifidobacteria reveals a higher BSH activity than lactobacilli. The fecal unconjugated bile acid and secondary bile acid levels in Subject A increased after the LGG supplementation (0.36-1.79 and 1.82-16.19 $\mu \mathrm{mol} / \mathrm{g}$ respectively). Although the LGG supplementation appears to promote bile acid deconjugation, most of the unconjugated bile acids in Subject A appear to have been modified into secondary bile acids. Alternatively, in Subject B there were no significant changes throughout the study.

Conclusion: We observed a significant increase in the fecal secondary bile acid levels after probiotic administration in one of our cases. Further studies are needed to elucidate the factors affecting $7 \alpha$-dehydroxylation of bile acids to confirm the safety of using probiotics.

Keywords: bile salt hydrolase; BSH; dihydroxylation; Bifidobacterium

\section{BACKGROUND}

Several animal and human studies have reported various health benefits of probiotic use, such as gastrointestinal health improvement [1], allergy prevention [2], and influenza virus infection prevention [3]. Recently, there has been an increased interest in the hypocholesterolemic effect of probiotics [4], and few clinical experiments have successfully revealed that probiotics can be used to control the lipid levels in humans $[5,6]$. Several mechanisms have been hypothesized to explain the hypocholesterolemic effect of probiotics. The most likely mechanism has been attributed to bile salt hydrolase (BSH) activity [7]. Alternatively, the undesirable effects of BSH activity that produce toxic secondary bile acids are concerning. However, the influence of probiotic intake on bile acid metabolism remains unclear.

BSH is an enzyme produced by the bacterial species of several genera associated with the gastrointestinal tract, and hydrolyzes conjugated bile acids into amino acid residues and unconjugated bile acids. A major study screening more than 300 bacterial strains reported that most 
Bifidobacterium and Lactobacillus strains that colonize the mammalian intestines reveal BSH activity, and in general bifidobacteria have higher BSH activity than lactobacilli [8]. Additionally, several strains of commercially available probiotic bacteria also exhibit BSH activity [9]. Therefore, alteration of the intestinal microflora by ingesting probiotics and/or a selection of probiotics possessing BSH activity may promote the hydrolysis of conjugated bile acids in the intestines. As unconjugated bile acids are less soluble and less efficiently reabsorbed from the intestinal lumen, a majority of these acids are excreted in the feces [7, 10]. Thus, probiotics may facilitate bile acid excretion, increase de novo synthesis of bile acids from cholesterol, and cause lower serum cholesterol levels.

Conversely, several reviews have described how microbial BSH activity could potentially be detrimental to the human host $[11,12]$. After deconjugation by $\mathrm{BSH}$, the unconjugated bile acids (but not conjugated bile acids) may be converted to secondary bile acids through the microbial bile acid $7 \alpha$-dehydroxylation pathway [13]. Some researchers assume that nearly all bile acids that escape the enterohepatic circulation are converted to secondary bile acids before excretion [14]. Several studies have reported that secondary bile acids cause DNA damage and are linked to colon carcinogenesis [14-16]. Moreover, secondary bile acids have been found in high concentrations in the fecal water of colon cancer patients [17]. Accordingly, the effects of probiotic intake on bile acid metabolism need to be explored for safety measures. Thus, in order to further understand the influence of probiotic intake on fecal bile acid levels, we analyzed the bile acid levels and microbiota in the human fecal samples after probiotic supplementation.

\section{METHODS}

\section{Patients}

Two patients hospitalized for dyslipidemia and schizophrenia (Subjects A and B) were enrolled in this study. The baseline characteristics of the subjects are presented in Table 1. Both subjects received an atypical antipsychotic drug and an intestinal drug (magnesium oxide), and Subject B received medication that increases the peristaltic colon activity in addition to a cholesterollowering medication. Both subjects gave their informed consent to participate in this study, and the ethics committee of Yamagata Prefectural Yonezawa University of Nutrition Sciences approved this study. The study protocol conformed to the tenets of the Declaration of Helsinki.

\section{Study design}

We used supplement sachets of powdered Lactobacillus rhamnosus GG (LGG) containing $14 \times$ $10^{10}$ bacteria per sachet. Both subjects consumed the supplement three times a day with meals for 
4 weeks, and further avoided the supplement for the subsequent 4 weeks. Fecal samples were collected at baseline and after 4 weeks (supplementation) and 8 weeks (no supplementation). Their diet and medication remained unaltered, and the subjects were instructed to avoid consumption of foods or supplements comprising other lactic acid bacteria or bifidobacteria during the study period. Furthermore, their defecation frequency remained the same during the study period.

Table 1. Clinical characteristics of the study subjects at baseline.

\begin{tabular}{lcc}
\hline & Subject A & Subject B \\
\hline Sex & Male & Female \\
Age, years & 61 & 76 \\
BMI, kg/m² & 29 & 26 \\
To-Cho, mg/dl & 185 & 201 \\
TG, mg/dl & 136 & 179 \\
Diet & & \\
$\quad$ Energy, kcal/day & 1600 & 1400 \\
$\quad$ Protein, g/day & 60 & 50 \\
$\quad$ Fat, g/day & 35 & 35 \\
$\quad$ Fiber, g/day & 16 & 16 \\
Frequency of defecation & Everyday & Everyday \\
Diarrhea & No & No \\
Duration of hospitalization, years & $5 \sim 10$ & $5 \sim 10$ \\
\hline
\end{tabular}

BMI, body mass index; To-Cho, total cholesterol; TG, triglyceride.

\section{Analysis}

Fecal samples were collected three times (at baseline, 4 weeks, and 8 weeks) and stored at $-80{ }^{\circ} \mathrm{C}$ until further analysis. Fecal microbiota was analyzed by targeting the bacterial 16S rRNA genes using the terminal restriction fragment length polymorphism (T-RFLP) [18-21]. The fecal concentrations of conjugated bile acids (glycocholic acid, taurocholic acid, glycochenodeoxycholic acid, and taurochenodeoxycholic acid), unconjugated bile acids (cholic acid and chenodeoxycholic acid), and secondary bile acids (deoxycholic acid and lithocholic acid) were measured using liquid chromatography/quadrupole time-of-flight mass spectrometry (LCQTOF/MS). All samples were analyzed by Techno Suruga Laboratory Co., Ltd. (Shizuoka, Japan). 


\section{RESULTS}

Alterations in the intestinal microflora may affect the bile acid deconjugation, because most Bifidobacterium and Lactobacillus strains that colonize the intestines exhibit BSH activity [8]. The relative abundance of Bifidobacterium and Lactobacillales in the feces was assessed by T-RFLP analysis (Figure 1). The relative abundance of Bifidobacterium, including strains that report high BSH activity [8], increased after probiotic supplementation in Subject A (30\%-49\%), and then decreased after the nonsupplementation period (to 35\%). Interestingly, the relative abundance of Bifidobacterium in Subject B remained relatively constant throughout the study period.

To examine the influence of probiotic intake on fecal bile acid levels, we analyzed these levels in fecal samples collected at baseline and after the supplementation and nonsupplementation periods (Figure 2). The fecal unconjugated bile acid and secondary bile acid levels in Subject A increased 5-fold (0.36-1.79 $\mu \mathrm{mol} / \mathrm{g})$ and 8.8-fold (1.82-16.19 $\mu \mathrm{mol} / \mathrm{g})$ after supplementation, respectively, and these levels decreased after the nonsupplementation period. Alternatively, the fecal bile acid levels in Subject B remained relatively constant throughout the study period, and no fecal unconjugated bile acids were detected.

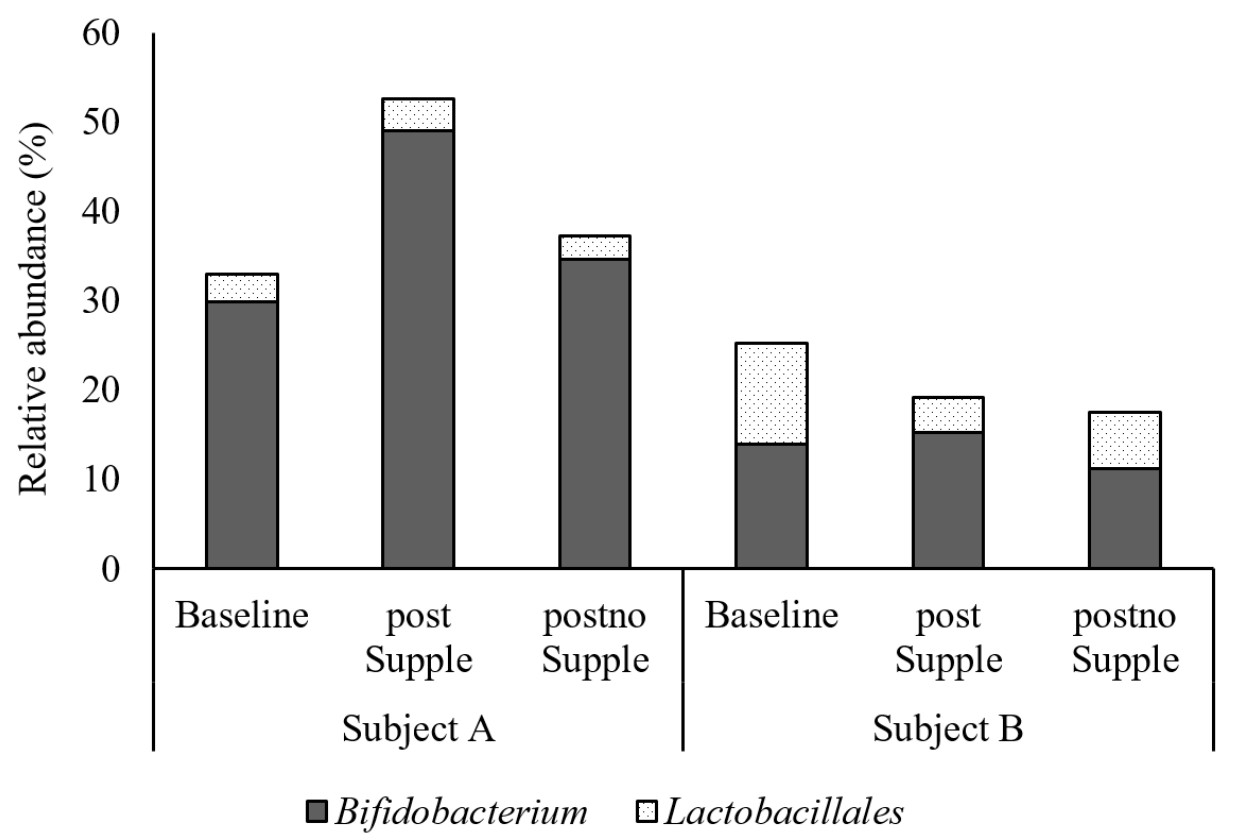

Figure 1. Changes in relative abundance of bacteria with BSH activity (Bifidobacterium and Lactobacillus) in fecal samples. Fecal samples were collected at baseline and after supplementation (postSupple) and non-supplementation (postnoSupple) periods. 


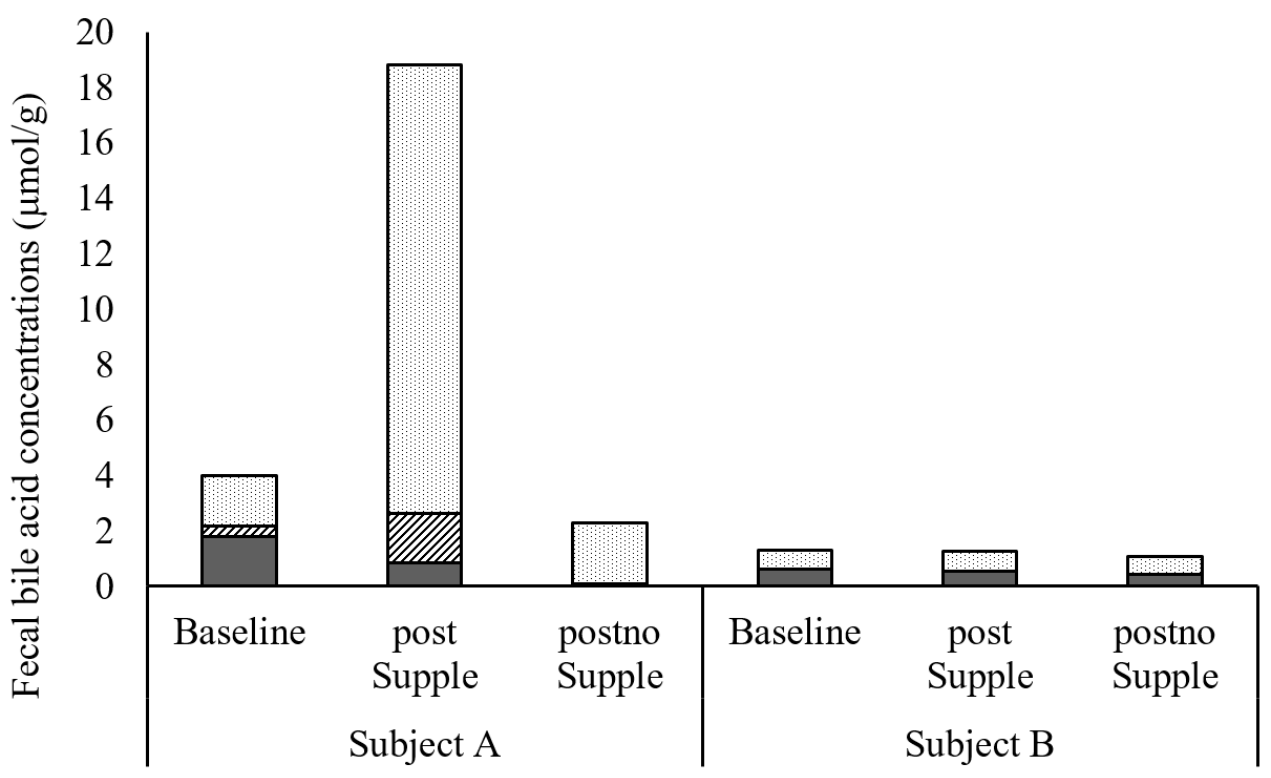

$\square$ Conjugated bile acids $\square$ Unconjugated bile acids $\square$ Secondary bile acids

Figure 2. Changes in bile acid concentrations in fecal samples. Fecal samples were collected at baseline and after supplementation (postSupple) and non-supplementation (postnoSupple) periods. Conjugated bile acids: glycocholic acid, taurocholic acid, glycochenodeoxycholic acid, and taurochenodeoxycholic acid. Unconjugated bile acids: cholic acid and chenodeoxycholic acid. Secondary bile acids: deoxycholic acid and lithocholic acid.

\section{DISCUSSION}

In Subject A, the bile acid deconjugation appeared to be promoted by probiotic supplementation. Accordingly, most of the unconjugated bile acids could be modified into secondary bile acids through the microbial bile acid $7 \alpha$-dehydroxylation pathway. This pathway is found in several species of Clostridium and Eubacterium [22]. Recent estimates indicate that this metabolic pathway is found in only $0.0001 \%$ of the total colonic flora, by species belonging to the Clostridium genus [23]. Kitahara et al. investigated the correlation between the count of Clostridium scindens and secondary bile acid levels in the fecal samples from 40 healthy Japanese subjects [24]. Although C. scindens is one of the most important bacterial species for bile acid $7 \alpha-$ dehydroxylating activity, no correlation was observed between the $C$. scindens count and secondary bile acid levels in the fecal samples. Moreover, they also analyzed the fecal microbiota using T-RFLP, and their results suggested that the intestinal microbiota has a stronger effect on the deoxycholic acid level than does the count of $C$. scindens cells. Marteau et al. observed that the fecal unconjugated bile acid concentration tended to increase after consuming Bifidobacteriumfermented milk. However, the secondary bile acid levels remained significantly unaffected, 
suggesting that the shortening of the colonic transit time was not due to secondary bile acid modifications [25]. In the present study, both subjects received an atypical antipsychotic drug, of which decreased peristaltic colon activity is a side effect. Subject B but not Subject A received medication which increases the peristaltic colon activity. Accordingly, there is a growing interest in the influence of decreasing peristaltic colon activity on bile acid metabolism.

Several limitations must be considered in interpreting our results. First, the design and number of patients of this study are insufficient. The elevated fecal secondary bile acid levels in this study at the point after taking probiotics may be related to food or other causes. A randomized, placebocontrolled crossover study is needed to explore whether probiotics are responsible for an increase in the fecal secondary bile acid levels. Additionally, various factors such as dietary patterns, composition of microbiota, and bowel pattern may affect fecal secondary bile acid levels. Such factors should be explored, and future trials should investigate to elucidate the factors affecting bile acid $7 \alpha$-dehydroxylation to confirm the safety of administering probiotics. Second, although intake of probiotic bacteria with BSH activity is considered as a major factor in promoting bile acid deconjugation, it is unclear whether LGG reveals BSH activity (sequences of putative conjugated BSH from LGG have been deposited in GenBank [NCBI]).

\section{CONCLUSION}

In conclusion, we report a case exhibiting increased levels of fecal secondary bile acids after receiving probiotics. Further studies are needed to elucidate the factors affecting bile acid $7 \alpha-$ dehydroxylation to confirm the safety of administering probiotics.

List of Abbreviations: BSH, bile salt hydrolase; LGG, Lactobacillus rhamnosus GG; T-RFLP, terminal restriction fragment length polymorphism.

Competing Interests: The authors declare that they have no competing interests.

Authors' Contributions: All authors equally contributed to this study.

Authors' Information: YS, Master's, Teaching Associate of Department of Health and Nutrition, Yamagata Prefectural Yonezawa University of Nutrition Sciences; HN, Registered Dietitian, Manager Section Chief of Department of Nutrition, Public Interest Incorporated Foundation Sekizen-kai Soga hospital; YK, Registered Dietitian, Manager Section Chief of Department of 
Nutrition, Medical Corporation, Ziundo hospital; TS, Master's, Associate Professor of Department of Health and Nutrition, Yamagata Prefectural Yonezawa University of Nutrition Sciences.

Acknowledgements and Funding: Fecal analysis and probiotic supplements were supported by Takanashi Milk Products Co., Ltd.

\section{REFERENCES}

1. Jones ML, Martoni CJ, Ganopolsky JG, Sulemankhil I, Ghali P, Prakash S: Improvement of gastrointestinal health status in subjects consuming Lactobacillus reuteri NCIMB 30242 capsules: a post-hoc analysis of a randomized controlled trial. Expert Opin Biol Ther 2013, 13(12):1643-1651.

2. Kalliomäki M, Salminen S, Arvilommi H, Kero P, Koskinen P, Isolauri E: Probiotics in primary prevention of atopic disease: a randomised placebo-controlled trial. Lancet 2001, 357(9262):1076-1079.

3. Nishihira J, Moriya T, Sakai F, Kabuki T, Kawasaki Y, Nishimura M: Lactobacillus gasseri SBT2055 stimulates immunoglobulin production and innate immunity after influenza vaccination in healthy adult volunteers. Functional Foods in Health and Disease 2016, 6(9):544-568.

4. Ishimwe N, Daliri EB, Lee BH, Fang F, Du G: The perspective on cholesterol-lowering mechanisms of probiotics. Mol Nutr Food Res 2015, 59(1):94-105.

5. Sadrzadeh-Yeganeh H, Elmadfa I, Djazayery A, Jalali M, Heshmat R, Chamary M: The effects of probiotic and conventional yoghurt on lipid profile in women. Br J Nutr 2010, 103(12):1778-1783.

6. Ataie-Jafari A, Larijani B, Alavi Majd H, Tahbaz F: Cholesterol-lowering effect of probiotic yogurt in comparison with ordinary yogurt in mildly to moderately hypercholesterolemic subjects. Ann Nutr Metab 2009, 54(1):22-27.

7. Lye H, Ramnat-Ali G, Liong M: Mechanisms of cholesterol removal by lactobacilli under conditions that mimic the human gastrointestinal tract. Int Dairy J 2010, 20(3):169-175.

8. Tanaka H, Doesburg K, Iwasaki T, Mierau I: Screening of lactic acid bacteria for bile salt hydrolase activity. J Dairy Sci 1999, 82(12):2530-2535.

9. Jones ML, Martoni CJ, Prakash S: Cholesterol lowering and inhibition of sterol absorption by Lactobacillus reuteri NCIMB 30242: a randomized controlled trial. Eur J 
Clin Nutr 2012, 66(11):1234-1241.

10. Huang Y, Wang X, Wang J, Wu F, Sui Y, Yang L, Wang Z: Lactobacillus plantarum strains as potential probiotic cultures with cholesterol-lowering activity. J Dairy Sci 2013, 96(5):2746-2753.

11. Pavlović N, Stankov K, Mikov M: Probiotics--interactions with bile acids and impact on cholesterol metabolism. Appl Biochem Biotechnol 2012, 168(7):1880-1895.

12. Begley M, Gahan CG, Hill C: The interaction between bacteria and bile. FEMS Microbiol Rev 2005, 29(4):625-651.

13. Batta AK, Salen G, Arora R, Shefer S, Batta M, Person A: Side chain conjugation prevents bacterial 7-dehydroxylation of bile acids. J Biol Chem 1990, 265(19):1092510928.

14. McGarr SE, Ridlon JM, Hylemon PB: Diet, anaerobic bacterial metabolism, and colon cancer: a review of the literature. J Clin Gastroenterol 2005, 39(2):98-109.

15. Glinghammar B, Inoue H, Rafter JJ: Deoxycholic acid causes DNA damage in colonic cells with subsequent induction of caspases, $C O X-2$ promoter activity and the transcription factors NF- $\kappa \mathrm{B}$ and AP-1. Carcinogenesis 2002, 23(5):839-845.

16. Kandell RL, Bernstein C: Bile salt/acid induction of DNA damage in bacterial and mammalian cells: implications for colon cancer. Nutr Cancer 1991, 16(3-4):227-238.

17. Bernstein H, Bernstein C, Payne CM, Dvorakova K, Garewal H: Bile acids as carcinogens in human gastrointestinal cancers. Mutat Res 2005, 589(1):47-65.

18. Takahashi S, Tomita J, Nishioka K, Hisada T, Nishijima M: Development of a prokaryotic universal primer for simultaneous analysis of bacteria and archaea using next-generation sequencing. PLoS One 2014, 9(8) e105592.

19. Nagashima K, Hisada T, Sato M, Mochizuki J: Application of new primer-enzyme combinations to terminal restriction fragment length polymorphism profiling of bacterial populations in human feces. Appl Environ Microbiol 2003, 69(2):1251-1262.

20. Nagashima K, Mochizuki J, Hisada T, Suzuki S, Shimomura K: Phylogenetic analysis of $16 \mathrm{~S}$ ribosomal RNA gene sequences from human fecal microbiota and improved utility of terminal restriction fragment length polymorphism profiling. Biosci Microflora 2006, 25(3):99-107.

21. Collins MD, Lawson PA, Willems A, Cordoba JJ, Fernandez-Garayzabal J, Garcia P, Cai J, Hippe H, Farrow JA: The phylogeny of the genus Clostridium: proposal of five new genera and eleven new species combinations. Int J Syst Bacteriol 1994, 44(4):812- 
826.

22. Doerner KC, Takamine F, LaVoie CP, Mallonee DH, Hylemon PB: Assessment of fecal bacteria with bile acid 7 alpha-dehydroxylating activity for the presence of bai-like genes. Appl Environ Microbiol 1997, 63(3):1185-1188.

23. Lefebvre P, Cariou B, Lien F, Kuipers F, Staels B: Role of bile acids and bile acid receptors in metabolic regulation. Physiol Rev 2009, 89(1):147-191.

24. Kitahara M, Sakata S, Sakamoto M, Benno Y: Comparison among fecal secondary bile acid levels, fecal microbiota and Clostridium scindens cell numbers in Japanese. Microbiol Immunol 2004, 48(5):367-375.

25. Marteau P, Cuillerier E, Meance S, Gerhardt MF, Myara A, Bouvier M, Bouley C, Tondu F, Bommelaer G, Grimaud JC: Bifidobacterium animalis strain DN-173 010 shortens the colonic transit time in healthy women: a double-blind, randomized, controlled study. Aliment Pharmacol Ther 2002, 16(3):587-593. 